\title{
Theoretical Assessment of an All-Optical Temporal Low-Pass Filter for Dynamic Fiber Bragg Grating Signals
}

\author{
T. C. Buck, M. S. Müller, and A. W. Koch \\ Institute for Measurement Systems and Sensor Technology, Technische Universität München, Theresienstraße 90/N5, \\ 80333 München, Germany \\ Correspondence should be addressed to T. C. Buck, t.buck@tum.de
}

Received 16 December 2010; Accepted 7 July 2011

Academic Editor: Ignacio Matias

Copyright ( $\odot 2011$ T. C. Buck et al. This is an open access article distributed under the Creative Commons Attribution License, which permits unrestricted use, distribution, and reproduction in any medium, provided the original work is properly cited.

Fiber Bragg grating (FBG) sensors are used in numerous applications. Currently, the assessment of dynamic loads with FBGs is of increasing interest. Those applications include vibration analysis, acceleration, or dynamic strain estimation. The acquisition of dynamic signals usually incorporates the application of signal low-pass filtering before signal quantization. FBG interrogators based on spectrometers or scanning lasers inherently have a limited antialiasing capability for dynamic FBG signals. We present a theoretical analysis of an all optical low-pass filter for spectrometer-based interrogators. The low-pass filter is based on the application of a luminescent material onto the detector. Light absorption and continuous light reemission lead to an advanced attenuation of high FBG signal frequencies. An analytic derivation of the system transfer function and numerical signal simulations are presented.

\section{Introduction}

Fiber-Bragg-grating- (FBG-) based measurement systems are attractive due to the inherent immunity of FBG sensors against electromagnetic interference, low sensor dimensions and weight, and the multiplexability of a plurality of FBG sensors within one sensing fiber [1]. Several interrogation methods have been developed and are already commercially available. Systems based on scanning laser spectral interrogators, edge-filter-based interrogators, scanning-filter-based approaches, and systems based on spectrometers are the most prominent implementations [2-5]. FBG sensors have been intensively employed in quasistatic environments. Currently, there is an increasing interest in the application of FBG sensors for the acquisition of dynamic loads [6]. Among those dynamic quantities to be measured are accelerations $[7,8]$, lamb wave propagation in composite structures [9], dynamic structural shape monitoring [10], and so forth. In general, the acquisition of dynamic loads requires a low-pass filtering of the signal before quantization and sampling. Insufficient signal low-pass filtering can lead to the occurrence of aliased signal components in the acquired signal [11]. FBG measurement systems incorporating high antialiasing capabilities based on spectral FBG signal filtering have been demonstrated [12, 13]. Those interrogators, however, offer a limited number of sensors per interrogator. FBG interrogators based on spectrometers exhibit a certain low-pass behaviour due to temporal signal integration before signal quantization [14]. However, this inherent low-pass is not comparable to standard low-pass filters employed in electric measurement systems.

We propose a method for application of an additional signal low-pass filter for the acquisition of dynamic loads using spectrometers.

\section{All-Optical FBG Low-Pass Filter}

Figure 1 exemplarily depicts the interrogation scheme of a spectrometer-based FBG interrogator. A FBG is illuminated by a broadband light source. A narrow wavelength band around the Bragg wavelength $\lambda_{B}$ is reflected and guided via an optical circulator to the spectrometer. The dispersing element in the spectrometer images the sensor spectrum linearly onto a CCD line array. 


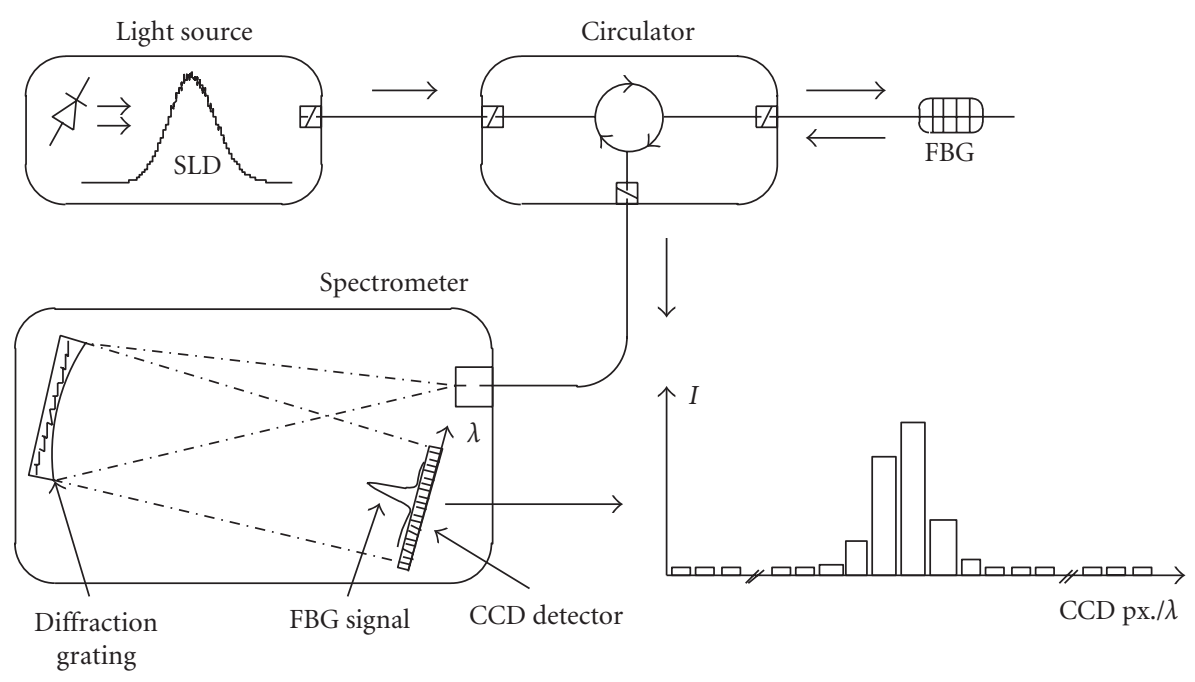

FIGURE 1: Schematic of a spectrometer-based FBG interrogator.

2.1. State of the Art. In conventional spectrometric FBG interrogation systems, the attenuation of high signal frequencies is determined by the temporal signal integration of the detector, as described by Van Damme et al. [14]. This lowpass behaviour is tuneable to certain extent by adjusting the fraction of the integration time compared to the overall cycle time of the sensor. The maximum performance, that is, the maximum signal amplitude attenuation at high signal frequencies, is achieved for a maximum integration time. The relative transfer function is then given by

$$
R(f)=\operatorname{sinc}\left(\frac{\pi f}{f_{s}}\right),
$$

where $f_{s}$ is the sampling frequency and $f$ is the signal frequency. This indicates a maximum signal attenuation of $3.98 \mathrm{~dB}$ at the Nyquist frequency $f_{\text {Nyquist }}=f_{s} / 2$. Signal components with $f>f_{\text {Nyquist }}$ will thus be detected as aliased frequency components with nonvanishing amplitude.

2.2. System Description. The proposed system implements a signal low-pass filter to spectrometric interrogators based on a continuous delay of the spectral signal on the detector. The continuous delay is accomplished by light absorption and continuous light reemission within a luminescent material. A possible arrangement of the proposed system is depicted in Figure 2. A luminescent material is placed on top of the light-sensitive detector. The layer absorbs incident light being refracted at the spectrometer grating and continuously reemits it towards the detector. A description of the low-pass sensing mechanism of the system is given in the following.

A FBG peak at a position $x_{0}\left(\lambda_{B}\right)$ with intensity $I_{0}$ on the detector at a time $t_{0}$ is absorbed within the layer on top of the detector. The light will be continuously reemitted at position $x_{0}\left(\lambda_{B}\right)$ with an intensity $I\left(t_{0}+t^{\prime}\right)=I^{\prime}{ }_{0} \cdot e^{-t^{\prime} / \tau}$ at time $t_{0}+t^{\prime}$, where $\tau$ is the lifetime of the transition in the luminescent material. Conclusively, a trend $\lambda_{B}(t)$ of a FBG peak will be continuously superimposed with a family of trends $\lambda_{B}(t-\Delta t)$

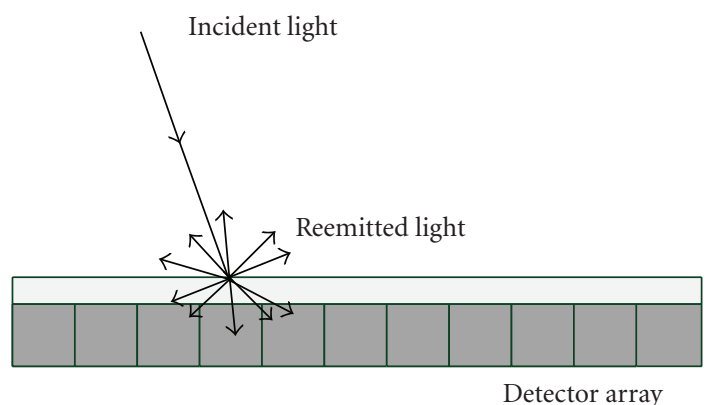

FIGURE 2: Sensor array covered with phosphorescent layer. Diffracted light from the spectrometer grating is focussed onto the sensor array. The light is absorbed and reemitted in the phosphorescent layer.

with intensity $I^{\prime}{ }_{0} \cdot e^{-\Delta t / \tau}, \Delta t>0[15]$. This description is valid for constant absorption rates in the material; that is, the probability of absorption for an incident photon must be constant. The signal generation process is exemplarily depicted in Figure 3. The original trend of the FBG peak on the detector is depicted by the black solid trend. The delayed attenuated signals are indicated by the grayed lines.

If the layer thickness is sufficiently small, smearing effects due to nondirectional light emission can be neglected. It is necessary that the detector array is sensitive for light in the spectral band of the reemitted light from the luminescent layer.

2.3. Transfer Function Estimation. The Bragg wavelength $\lambda_{B}$ of the sensor is given by the center of gravity (COG) of the spectral sensor signature [16]. From a reflection spectrum $I(\lambda)$, the Bragg wavelength is given by

$$
\lambda_{B}=\frac{\int_{\lambda=0}^{\lambda=\infty} \lambda \cdot I(\lambda) d \lambda}{\int_{\lambda=0}^{\lambda=\infty} I(\lambda) d \lambda},
$$




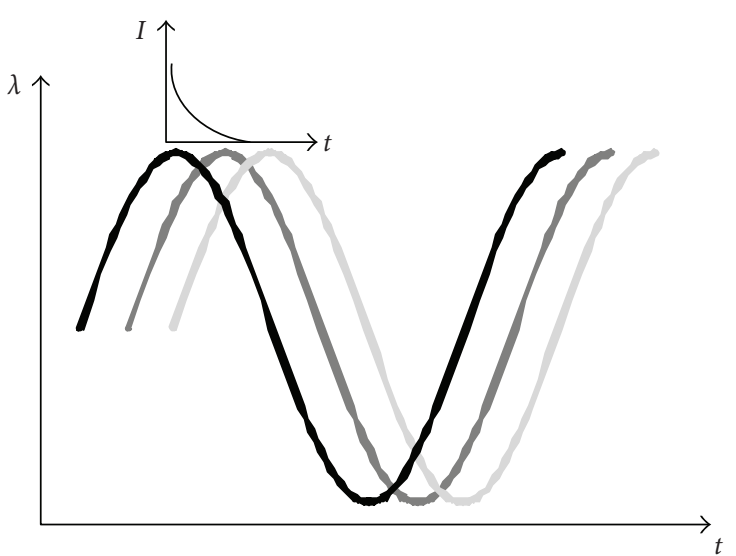

FIGURE 3: Illustration of signal generation process. A signal trend on the detector is smeared in time by afterglowing in the phosphorescent layer. The intensity variation of the reemitted light is depicted by light colour.

where $I(\lambda)$ is the spectral intensity of the FBG peak. The spectral FBG reflection at time $t_{0}$ is assumed to be a delta distribution at the Bragg wavelength $\lambda_{B}, I\left(\lambda, t_{0}\right)=\delta(\lambda-$ $\left.\lambda_{B}\left(t_{0}\right)\right)$. The cumulated light intensity on the detector at wavelength $\lambda$ is thus given by $I_{\text {int }}\left(\lambda, t_{0}\right)=\int_{t=-\infty}^{t=0} \delta(\lambda-$ $\left.\lambda_{B}\left(t^{\prime}\right)\right) e^{t^{\prime} / \tau} d t^{\prime}$, where $\tau$ is the lifetime of the transition in the luminescent layer. Considering a sinusoidal Bragg wavelength trend $\lambda_{B}=\sin (\omega t)$ at time $t_{0}$, the nominator in (2) can be expressed as

$$
\begin{aligned}
\int_{\lambda=0}^{\lambda=\infty} \lambda \cdot I_{\text {int }}\left(\lambda, t_{0}\right) d \lambda & =\int_{t=-\infty}^{t=0} \sin \left(\omega\left(t_{0}+t^{\prime}\right)\right) e^{t^{\prime} / \tau} d t^{\prime} \\
& =-\frac{\tau\left(\omega \tau \cos \left(\omega t_{0}\right)-\sin \left(\omega t_{0}\right)\right)}{\omega^{2} \tau^{2}+1} .
\end{aligned}
$$

The denominator integral can be simplified accordingly to

$$
\int_{\lambda=0}^{\lambda=\infty} I_{\text {int }}(\lambda) d \lambda=\int_{t^{\prime}=-\infty}^{t^{\prime}=0} e^{t^{\prime} / \tau} d t^{\prime}=\tau .
$$

Consequently, the COG at time $t$ of a sinusoidal Bragg wavelength trend $\lambda_{B}=\sin (\omega t)$ for a spectrometric system incorporating a luminescent layer is given by

$$
\lambda_{B, \mathrm{COG}}(t)=-\frac{(\omega \tau \cos (\omega t)-\sin (\omega t))}{\omega^{2} \tau^{2}+1} .
$$

The frequency spectrum of this trend is given by

$$
F(\omega, \tau)= \pm \frac{\omega \tau}{\omega^{2} \tau^{2}+1}-\mp \frac{i}{\omega^{2} \tau^{2}+1} .
$$

The amplitude transfer function of the COG trend is thus given by

$$
|F(\omega, \tau)|=\frac{1}{\sqrt{\omega^{2} \tau^{2}+1}}
$$

with a $-3 \mathrm{~dB}$ cutoff frequency of $f_{-3 \mathrm{~dB}}=1 / 2 \pi \tau$. This derivation shows that the proposed system exhibits a lowpass characteristic for sinusoidal Bragg-wavelength trends.
The attenuation of high signal frequencies is dependent on the decay time of the excited electronic state of the phosphorescent layer on the detector array. Consequently, this all-optical low-pass filtering can be tuned to a certain cutoff frequency independent from the readout frequency of the detector.

\section{Total Relative Transfer Function}

Temporal signal integration on the detector gives rise to an inherent low-pass filter of spectrometric detection systems for FBG sensors. In general, the relative transfer function of such a system without luminescent layer is given by

$$
R(f)=\delta \cdot \operatorname{sinc}\left(\delta \frac{\pi f}{f_{s}}\right)
$$

where $f_{s}$ is the readout frequency, of the sensor, $f$ is the signal frequency, and $\delta$ is the fraction of signal integration time during one readout cycle [14]. In the proposed setup the signal on the detector as derived in (5) is integrated on the detector. The relative transfer function of the proposed system can thus be calculated by

$$
\left|R_{\text {total }}(f)\right|=|F(f, \tau) \cdot R(f)|,
$$

giving

$$
\left|R_{\text {total }}(f)\right|=\frac{\delta}{\sqrt{4 \pi^{2} f^{2} \tau^{2}+1}}\left|\operatorname{sinc}\left(\delta \frac{\pi f}{f_{s}}\right)\right| .
$$

\section{Numerical Model Validation}

The proposed system is modeled by a numerical simulation using MATLAB. The spectral trend on the detector is simulated with a time resolution of $d t=10^{-5} \mathrm{~s}$, and the lifetime of the phosphorescent material is set to $\tau=5 \cdot 10^{-3} \mathrm{~s}$. The simulated FBG peak exhibits a Gaussian profile with a FWHM of three pixels on the detector plane. Figure 4 shows the transfer function according to (7) and the relative transfer function extracted from the numerically simulated signal data without signal integration by the detector. The evaluated low-pass filter exhibits a roll off of about $-10 \mathrm{~dB}$ per decade. The extracted relative transfer function from the numerically simulated data is in good agreement with the calculated transfer function. The derived transfer function for a delta-shape FBG is a good model for FBGs with a Gaussian shape. As expected, the amplitude of the FBG COG trend rapidly decreases for high signal frequencies.

Figure 5 shows the total amplitude transfer function of the spectrometric interrogator system including signal integration and averaging on the detector array at a readout frequency $f_{s}=500 \mathrm{~Hz}$ with $\delta=1$ for different phosphorescence life times from $\tau=2 \mathrm{~ms}$ to $10 \mathrm{~ms}$. The transfer function of a spectrometric interrogator without phosphorescent layer exhibits a first root at $f=f_{s}$. Signals above the Nyquist frequency $f_{\text {Nyquist }}=250 \mathrm{~Hz}$ are not attenuated sufficiently for providing strong antialiasing capability. The acquisition of dynamic signals requires low-pas filtering of a signal before 


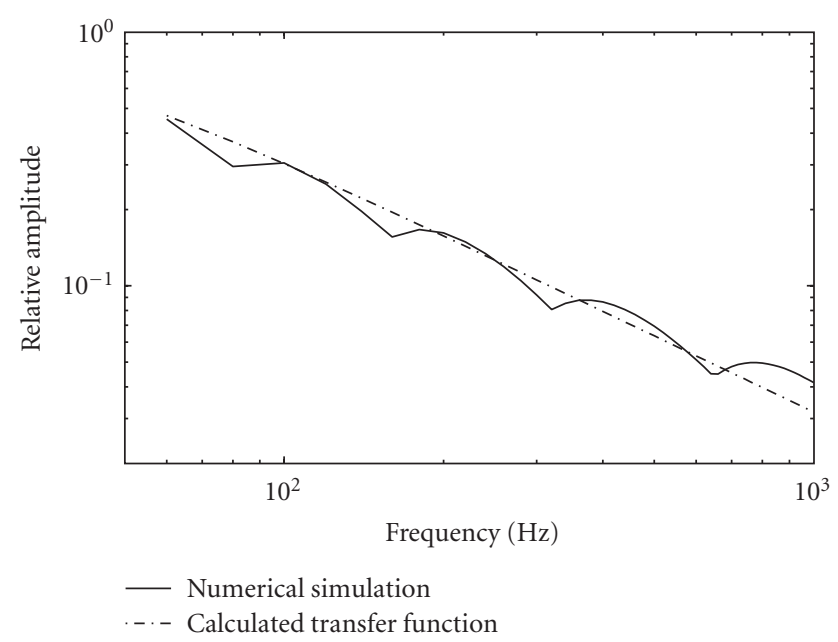

FIGURE 4: Calculated transfer function of a phosphorescent layer according to (7) (dotted line) and transfer function evaluated from numerically simulated FBG spectra (solid line). The lifetime of the phosphorescent transition is set to $5 \mathrm{~ms}$.

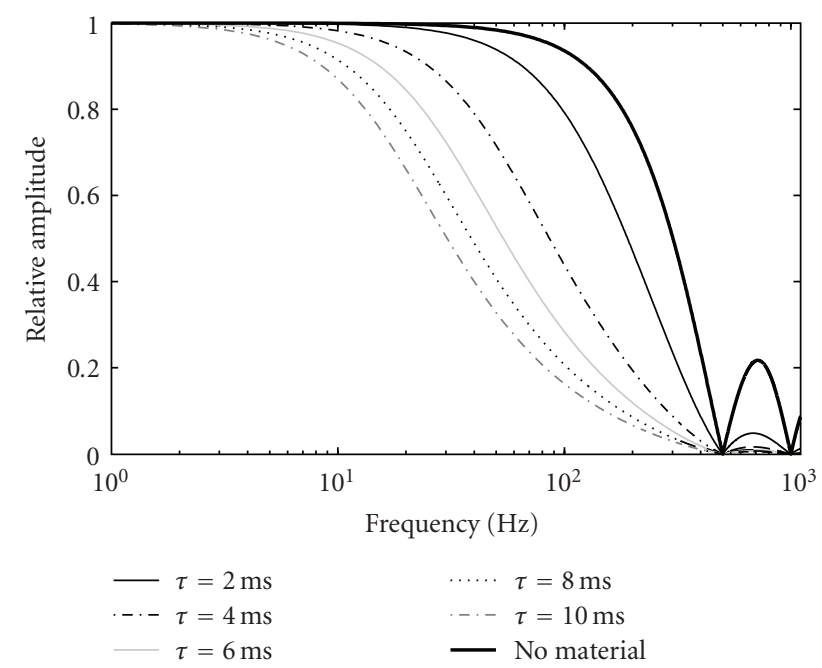

FIGURE 5: Calculated transfer functions for the proposed system with different configurations according to (10). An increasing low-pass behaviour for increasing decay times of the luminescent material is observable.

signal quantization. This is equal to an attenuation of signal frequencies above $f_{\text {Nyquist }}=250 \mathrm{~Hz}$. For increasing life times of the phosphorescent transition, the system cutoff frequency is shifted towards lower signal frequencies, providing an even stronger antialiasing capability.

\section{Conclusion}

We present a theoretical assessment of an all-optical low-pass for the spectrometric interrogation of dynamic FBG signals. The implementation of a luminescent layer in the optical path of the spectrometer in front of the detector acts as a continuous signal integrator. The derived analytic system transfer function is in good agreement with numerically simulated FBG signals. The cutoff frequency of the proposed low-pass filter is defined by the life time of the phosphorescent transition. For an implementation in a demonstrator system, luminescent dyes with high absorption coefficients at the FBG wavelength with sufficiently high optical decay time need to be identified. For FBG sensors around $1550 \mathrm{~nm}$, highly Erbium-doped glasses might be promising candidates [17]. Another promising material system could be given by materials that exhibit a NIR to VIS upconversion [18, 19], where transition lifetimes in the range of several ms have been observed.

\section{References}

[1] Y. J. Rao, "In-fibre Bragg grating sensors," Measurement Science and Technology, vol. 8, no. 4, pp. 355-375, 1997.

[2] B. Lee, "Review of the present status of optical fiber sensors," Optical Fiber Technology, vol. 9, no. 2, pp. 57-79, 2003.

[3] K. Schroeder, W. Ecke, E. Lembke, and G. Lenschow, "A fibre Bragg grating sensor system monitors operational load in a wind turbine rotor blade," Measurement Science and Technology, vol. 17, no. 5, pp. 1167-1172, 2006.

[4] S. M. Melle, K. Liu, and R. M. Measures, "A passive wavelength demodulation system for guided-wave Bragg grating sensors," IEEE Photonics Technology Letters, vol. 4, no. 5, pp. 516-518, 1992.

[5] S. H. Yun, D. J. Richardson, and B. Y. Kim, "Interrogation of fiber grating sensor arrays with a wavelength-swept fiber laser," Optics Letters, vol. 23, no. 11, pp. 843-845, 1998.

[6] J. R. Lee, C. Y. Ryu, B. Y. Koo, S. G. Kang, C. S. Hong, and C. G. Kim, "In-flight health monitoring of a subscale wing using a fiber Bragg grating sensor system," Smart Materials and Structures, vol. 12, no. 1, pp. 147-155, 2003.

[7] M. S. Müller, T. C. Buck, and A. W. Koch, "Fiber Bragg gratingbased acceleration sensor," in Proceedings of the International Symposium on Optomechatronic Technologies (ISOT '09), pp. 127-132, September 2009.

[8] X. F. Huang, Y. Liu, D. Luo et al., "Wavelet analysis on vibration modal frequency measurement at a low level of strain of the turbine blade using FBG sensors," Measurement Science and Technology, vol. 21, no. 1, Article ID 015305, 2010.

[9] C. S. Shin, B. L. Chen, and S. K. Liaw, "An FBG-based impact event detection system for structural health monitoring," Advances in Civil Engineering, vol. 2010, 2010.

[10] U. C. Mueller, H. Baier, T. Zeh, M. S. Mueller, and A. W. Koch, "Vibration and shape control in opto-mechanical systems using distributed fiber-optic bragg grating sensors," Journal of Vibration and Control, vol. 16, no. 4, pp. 539-553, 2010.

[11] R. L. Allen and D. W. Mills, Signal Analysis: Time, Frequency, Scale, and Structure, IEEE Press and Wiley-Interscience, Hoboken, NJ, USA, 2004.

[12] J. Bentell, D. Uwaerte, J. Cloots et al., " $250 \mathrm{khz}$ sampling rate fbg interrogator with strong anti-aliasing signal processing," in Proceedings of the 20th International Conference on Optical Fibre Sensors, 2009.

[13] D. C. Betz, G. Thursby, B. Culshaw, and W. J. Staszewski, "Acoustoultrasonic sensing using fiber bragg gratings," Smart Materials and Structures, vol. 12, p. 122, 2004.

[14] S. Van Damme, B. Boons, J. Vlekken, J. Bentell, and J. Vermeiren, "Dynamic fiber optic strain measurements and aliasing suppression with a PDA-based spectrometer," Measurement Science and Technology, vol. 18, no. 10, pp. 32633266, 2007. 
[15] C. Ronda, Luminescence: From Theory to Applications, WILEYVCH, Weinheim, Germany, 2008.

[16] T. Bodendorfer, M. S. Müller, F. Hirth, and A. W. Koch, "Comparison of different peak detection algorithms with regards to spectrometic fiber bragg grating interrogation systems," in Proceedings of the International Symposium on Optomechatronic Technologies, 2009.

[17] William J. Miniscalco, "Erbium-doped glasses for fiber amplifiers at 1500 nm," Journal of Lightwave Technology, vol. 9, no. 2, pp. 234-250, 1991.

[18] J. F. Suyver, A. Aebischer, D. Biner et al., "Novel materials doped with trivalent lanthanides and transition metal ions showing near-infrared to visible photon upconversion," Optical Materials, vol. 27, no. 6, pp. 1111-1130, 2005.

[19] S. Ivanova and F. Pellé, "Strong $1.53 \mu \mathrm{m}$ to NIR-VIS-UV upconversion in Er-doped fluoride glass for high-efficiency solar cells," Journal of the Optical Society of America B, vol. 26, no. 10, pp. 1930-1938, 2009. 

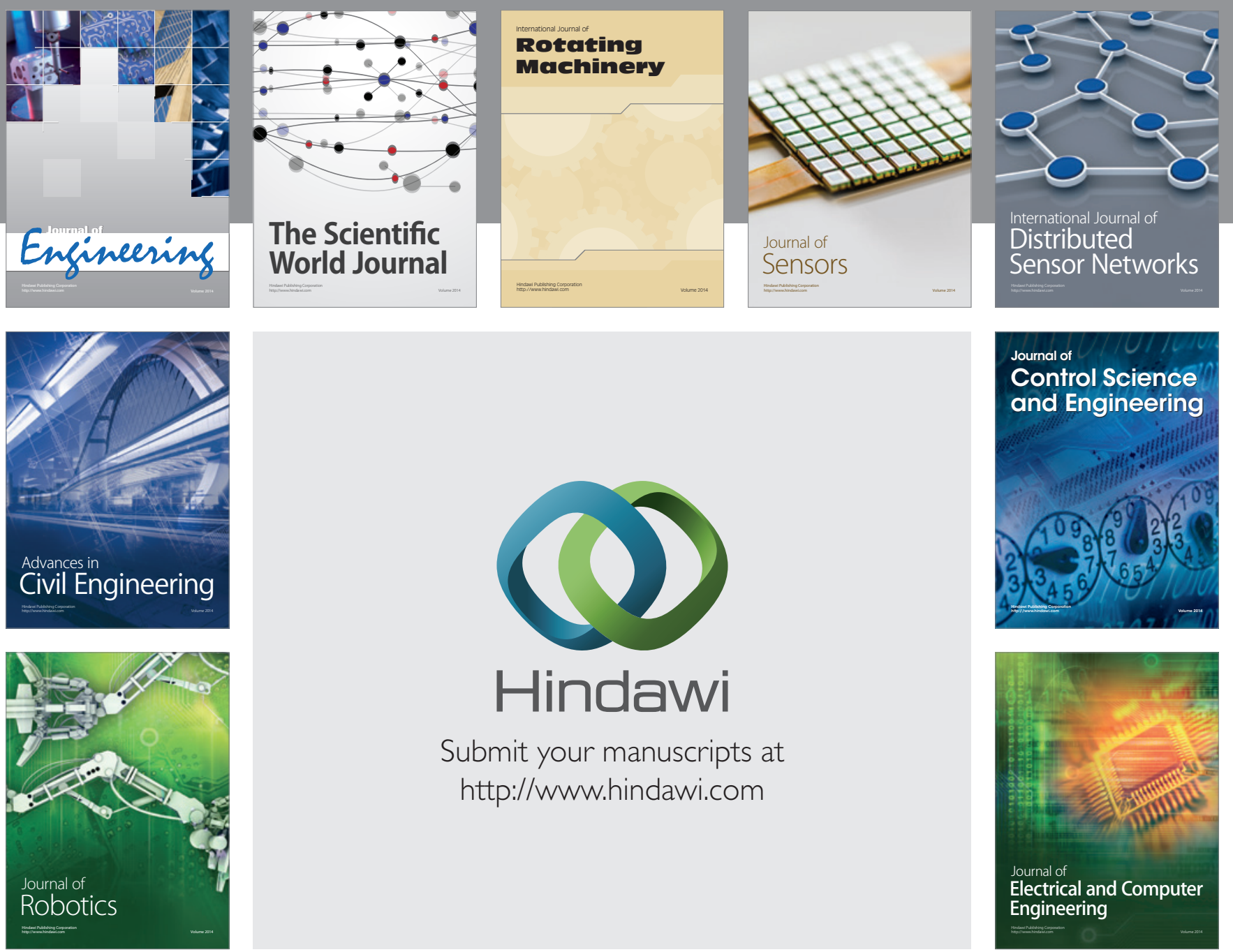

Submit your manuscripts at

http://www.hindawi.com
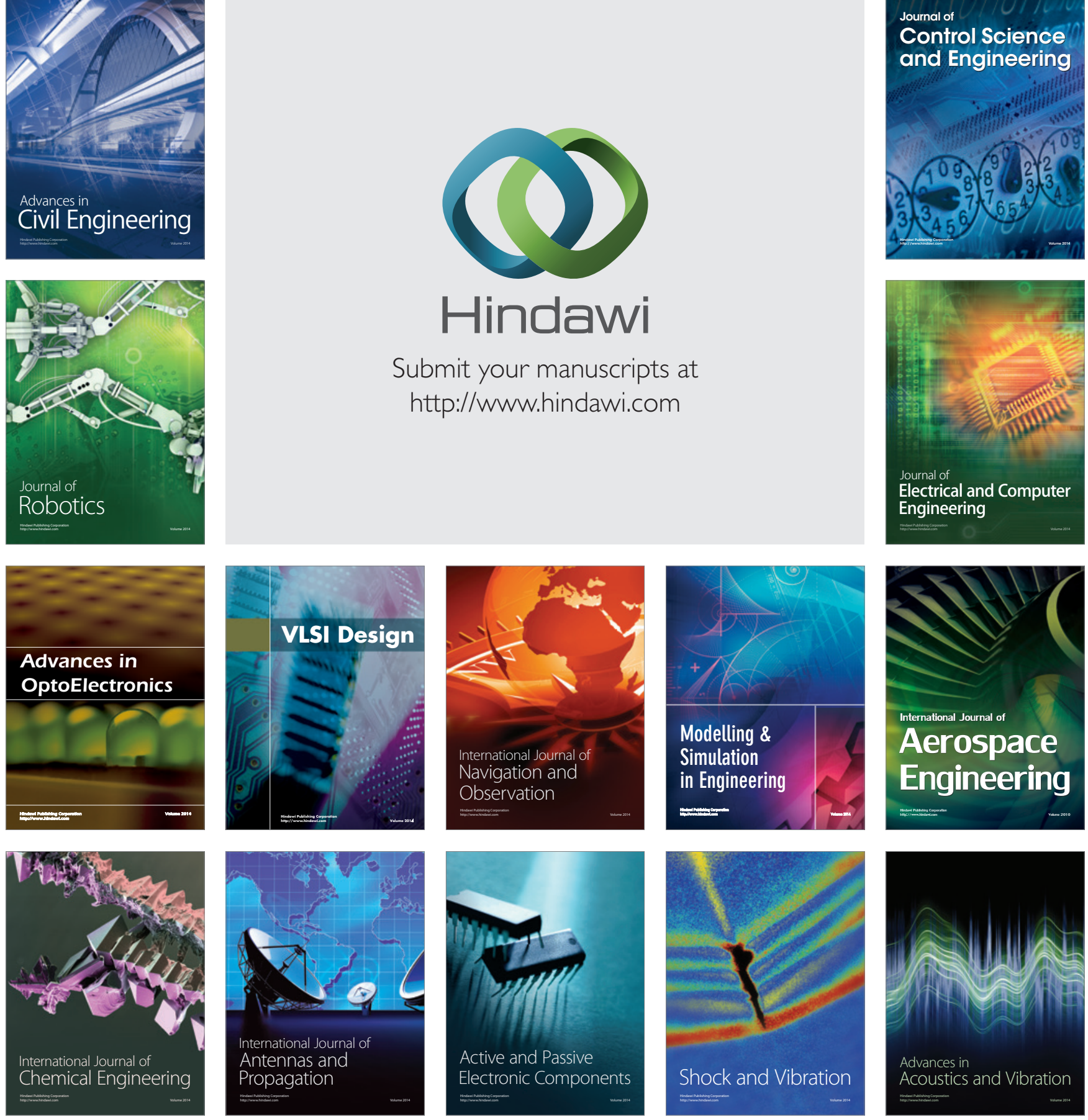